\title{
Determinación de la Concentración Óptima de Tres Crioprotectores para la Criopreservación de Espermatozoides Epididimarios de Alpaca
}

\author{
Determination of the Optimum Concentration of Three Cryoprotectants \\ for the Criopreservation of AlpaCa Epididymal Spermatozoa
}

\author{
Katherine Choez A. ${ }^{1,4}$, Luis Ruiz G. ${ }^{1}$, Rocío Sandoval M. ${ }^{1}$, Shirley Evangelista V. ${ }^{3}$, \\ Alexei Santiani A. ${ }^{2}$
}

\section{RESUMEN}

\begin{abstract}
El objetivo del presente estudio fue determinar la concentración óptima de glicerol, etilenglicol y dimetil sulfóxido (DMSO) en la criopreservación de espermatozoides epididimarios de alpaca. En el experimento 1 se evaluó el efecto de ocho concentraciones de glicerol, etilenglicol y DMSO: T1 (0 M), T2 (0.25 M), T3 (0.5 M), T4 (0.75 M), T5 (1 M), T6 $(1.25 \mathrm{M})$, T7 $(1.5 \mathrm{M})$ y T8 $(1.75 \mathrm{M})$. Se utilizó un modelo de regresión polinomial quíntuple para determinar la mejor concentración de cada crioprotector, resultando concentraciones óptimas para glicerol, etilenglicol y DMSO de 1.16, 1.02 y $0.9 \mathrm{M}$, respectivamente. En el Experimento 2, se comparó el efecto de las concentraciones óptimas entre glicerol, etilenglicol y DMSO sobre la motilidad, integridad de membrana plasmática y viabilidad e integridad acrosomal en los espermatozoides epididimarios de alpaca. Los resultados mostraron promedios de $35.8,51.8$ y $38.3 \%$ para DMSO $(0.9 \mathrm{M})$ y de de 30.8 , 45.0 y $33.8 \%$ para glicerol para las variables motilidad, integridad de membrana plasmática $\mathrm{y}$ viabilidad e integridad acrosomal, respectivamente, y sin diferencias significativas entre estos crioprotectores. Sin embargo, fueron significativamente mejores $(p<0.05)$ que para etilenglicol (1.02 M). Se concluye que las concentraciones de 0.9 M de DMSO y 1.16 $\mathrm{M}$ de glicerol conservaron mejor las características funcionales de los espermatozoides epididimarios de alpaca en comparación con etilenglicol (1.02 M).
\end{abstract}

Palabras clave: espermatozoide; epidídimo; alpaca; criopreservación

\footnotetext{
${ }^{1}$ Clínica de Animales Mayores, ${ }^{2}$ Laboratorio de Reproducción Animal, Facultad de Medicina Veterinaria, Universidad Nacional Mayor de San Marcos, Lima, Perú

${ }^{3}$ Laboratorio de Biotecnologías Reproductivas y Celulares, Facultad de Ciencias Veterinarias y Biológicas, Universidad Cientifica del Sur, Lima, Perú

${ }^{4}$ E-mail: kathyvet3@hotmail.com
}

Recibido: 27 de noviembre de 2016

Aceptado para publicación: 18 de marzo de 2017 
The aim of this study was to determine the optimal concentration of glycerol, ethylene glycol and dimethyl sulfoxide (DMSO) in the cryopreservation of epididymal spermatozoa of alpaca. The effect of different concentrations of glycerol, ethylene glycol and DMSO: T1 (0 M), T2 (0.25 M), T3 (0.5 M), T4 (0.75 M), T5 (1 M), T6 (1.25 M), T7 (1.5 M) and T8 $(1.75 \mathrm{M})$ was evaluated. A polynomial regression model was used to determine the best concentration of each cryoprotectant, resulting 1.16, 1.02 and $0.9 \mathrm{M}$ the optimal concentrations for glycerol, ethylene glycol and DMSO respectively. In Experiment 2, the effect of the optimal concentrations between glycerol, ethylene glycol and DMSO on motility, plasma membrane integrity, and acrosomal viability and integrity in alpaca epididymal spermatozoa were analyzed. The results showed a mean of 35.8, 51.8 and $38.3 \%$ for DMSO and 30.8, 45.0 and $33.8 \%$ for glycerol for motility, plasma membrane integrity and acrosome viability and integrity, respectively, and without significant differences between these cryoprotectants. However, the results were significantly better $(\mathrm{p}<0.05)$ than for ethylene glycol $(1.02 \mathrm{M})$. It is concluded that the concentrations of 0.9 $\mathrm{M}$ DMSO and $1.16 \mathrm{M}$ glycerol better preserved the functional characteristics of alpaca epididymal spermatozoa as compared to ethylene glycol (1.02 M).

Key words: spermatozoa; epididymis; alpaca; cryopreservation

\section{INTRODUCCIÓN}

Los estudios sobre conservación de semen fresco, refrigerado y congelado de camélidos sudamericanos (CSA), para su empleo en inseminación artificial, no ha obtenido el éxito esperado como en otras especies domésticas. Esto podría deberse a las características particulares que presenta el semen de los CSA, principalmente la alta viscosidad, bajo porcentaje de motilidad y baja concentración espermática (Bravo et al., 1997). Estas características espermáticas han condicionado la evaluación, manipulación, dilución y conservación del semen en esta especie (Tibary y Memon, 1999).

Los espermatozoides epididimarios constituyen un modelo experimental para el desarrollo de los trabajos de conservación de semen, ya que se encuentran en un nivel de maduración final y no están mezclados con el plasma seminal viscoso (Canorio, 2008).

Se dispone de diversos trabajos de investigación sobre criopreservación de espermatozoides epididimarios, pero los re- sultados pos-descongelamiento, como la motilidad, son muy variables y, por lo general, deficientes, obteniéndose valores que varían entre 0 y 34\% (Morton et al., 2007, 2010; Canorio, 2008; Banda et al., 2010; Terreros et al., 2012) a partir de motilidades iniciales de $30-70 \%$. La escasa supervivencia de los espermatozoides de CSA al proceso de criopreservación podría deberse a la susceptibilidad inherente de la especie, los componentes de los dilutores y la producción de especies reactivas de oxígeno (ROS), los cuales han sido identificados en otras especies (Medeiros et al., 2002).

Asimismo, existe una gran limitación en lo concerniente al desarrollo de protocolos adecuados para la conservación de espermatozoides de CSA (dilutores, temperatura de equilibrio, velocidad de congelación y descongelación, entre otros). Los crioprotectores son componentes importantes de los dilutores, pues permiten mantener una mayor proporción de agua líquida a bajas temperaturas $y$, en consecuencia, una menor concentración de electrolitos, posibilitando la supervivencia celular durante el proceso de criopreservación (Stornelli et al., 2005). 
E1 crioprotector más utilizado en espermatozoides y semen de alpacas es el glicerol; el cual ha sido empleado en concentraciones de 2-7\% (0.27-0.96 M) (Santiani et al., 2005; Banda et al., 2010; Morton et al., 2010). Asimismo, se ha utilizado el etilenglicol en concentraciones de 1-7\% (0.21.25 M) (Santiani et al., 2005; Banda et al., 2010; Terreros et al., 2012) y dimetil sulfóxido (DMSO) en concentraciones de $0.9-7 \%$ (0.125-0.98 M) (Canorio, 2008; Terreros et al., 2012). Sin embargo, no se ha establecido el agente crioprotector y la concentración que ofrece mejores resultados posdescongelamiento del semen criopreservado. Por ello, el objetivo del presente estudio fue evaluar tres agentes crioprotectores (glicerol, etilenglicol y DMSO) y determinar las concentraciones óptimas para la adecuada motilidad espermática, la integridad funcional de la membrana plasmática y la viabilidad e integridad de la membrana acrosomal de los espermatozoides de alpaca.

\section{MATERIALES y Métodos}

\section{Lugar y Muestra de Estudio}

El estudio se realizó en el Laboratorio de Reproducción Animal de la Facultad de Medicina Veterinaria de la Universidad Nacional Mayor de San Marcos (Lima, Perú). Se utilizaron 27 epidídimos obtenidos del Camal Municipal de Huancavelica. Los epidídimos para los tres primeros experimentos fueron colectados entre marzo y julio de 2012, y los del experimento 4 entre marzo y julio de 2014.

Los epidídimos provenían de alpacas machos adultos, con más de 3 años de edad. Las dimensiones testiculares fueron de 3.5$4 \mathrm{~cm}$ de largo y $2-2.5 \mathrm{~cm}$ de ancho. Solo se utilizaron aquellos con una concentración mayor a $70 \times 10^{6}$ espermatozoides por mililitro y motilidad mayor o igual al $60 \%$. La muestra de estudio estuvo constituida por 168 muestras procedentes de 27 epidídimos.

\section{Recuperación de Espermatozoides Epi- didimarios}

La colección de los testículos/epidídimos se realizó inmediatamente después del sacrificio del animal. Los testículos fueron liberados de la túnica albugínea y se colocaron en un recipiente con $\mathrm{ClNa} 0.9 \%$ a $5{ }^{\circ} \mathrm{C}$. El tiempo transcurrido entre la colección y su llegada al laboratorio fue de 20 horas.

La separación del testículo de la cola del epidídimo se hizo en el laboratorio. Los epidídimos fueron lavados con $\mathrm{ClNa} 0.9 \%$. La recuperación de los espermatozoides epididimarios se realizó en una placa Petri a través de cortes seriados con un bisturí en la superficie de la cola epididimaria (Banda et al., 2010). Durante este proceso, se agregó $1.5 \mathrm{ml}$ del dilutor leche descremada - yema de huevo - fructosa (Santiani et al., 2005) a $37^{\circ} \mathrm{C}$ sobre la cola epididimaria para facilitar la salida de los espermatozoides.

El líquido de la placa Petri fue colocado en una estufa a $37^{\circ} \mathrm{C}$ por $5 \mathrm{~min}$. Luego, se tomó $0.5 \mathrm{ml}$ para evaluar las características espermáticas iniciales y $1.0 \mathrm{ml}$ se mezcló con $3 \mathrm{ml}$ del dilutor. Los $4 \mathrm{ml}$ resultantes se distribuyeron equitativamente en 8 alicuotas de 0.5 $\mathrm{ml}$ para cada tratamiento del experimento 1 . Para el experimento 2 se siguió el mismo procedimiento, con la diferencia de que la dilución final con el dilutor fue distribuida equitativamente en cuatro tratamientos de $1 \mathrm{ml}$.

\section{Diseño Experimental}

El objetivo del experimento 1 fue determinar la concentración óptima del glicerol, etilenglicol y DMSO.

Por ello, se trabajó con 120 muestras procedentes de quince epididimos. Esto significa que los espermatozoides epididimarios recuperados de un epidídimo se dividieron en 8 tratamientos de $0.5 \mathrm{ml}$. Los tratamientos con base a los tres crioprotectores fueron: T1 (0 M), T2 (0.25 M), T3 (0.50 M), T4 (0.75 M), T5 (1.00 M), T6 (1.25 M), T7 (1.50 M) y T8 $(1.75 \mathrm{M})$ 
El objetivo del experimento 2 fue evaluar las concentraciones óptimas resultantes del experimento 1. Se trabajó con 48 muestras procedentes de 12 epidídimos. Es decir, los espermatozoides epididimarios recuperados de un epidídimo se dividieron en 4 tratamientos de $1 \mathrm{ml}$. Los tratamientos con base a los tres crioprotectores fueron: $\mathrm{T} 1: \sin$ crioprotector; T2: concentración óptima de glicerol, T3: concentración óptima de etilenglicol; T4 concentración óptima de DMSO.

\section{Criopreservación y Descongelación}

El proceso de criopreservación consistió en la curva de enfriamiento y congelación. Para la curva de enfriamiento, las alícuotas de semen diluido de cada tratamiento fueron colocadas en tubos de vidrio dentro de un recipiente con agua a $35^{\circ} \mathrm{C}$, e introducido en una refrigeradora para bajar la temperatura hasta $5{ }^{\circ} \mathrm{C}$ en un tiempo aproximado de 150 minutos (tasa de enfriamiento: $1{ }^{\circ} \mathrm{C} / 5 \mathrm{~min}$ ). Luego se agregó el crioprotector respectivo (glicerol, etilenglicol o DMSO) y se dejó reposar por $30 \mathrm{~min}$, antes del llenado de pajillas de $0.25 \mathrm{ml}$. Se obtuvieron 2 pajillas por cada tratamiento en el experimento 1 ( $n=240$ pajillas) y en el experimento 2 se obtuvieron 4 pajillas por tratamiento $(n=192$ pajilllas).

Para la congelación, las pajillas fueron expuestas a vapores de nitrógeno en una caja de poliestireno expandido (tecnopor) por 20 min, para luego sumergirlas directamente al nitrógeno líquido. Las pajillas fueron almacenadas en el tanque de nitrógeno por siete días. La descongelación se realizó sumergiendo cada pajilla en baño maría a $37^{\circ} \mathrm{C}$ por $1 \mathrm{~min}$.

\section{Evaluación de los Espermatozoides}

Se evaluó la totalidad de las pajillas. Para la evaluación de los espermatozoidesepididimarios del experimento 1 se determinó la motilidad (Ax et al., 2000), mientras que en el experimento 2 , se evaluaron, ade- más, la integridad funcional de membrana espermática mediante la prueba hipoosmótica (Jeyendran et al., 1984) y la viabilidad e integridad acrosomal mediante la técnica de doble tinción azul tripán / giemsa (Didión et al., 1989).

En el caso de la prueba hipoosmótica, se consideró como Host + a espermatozoides con cola enrrollada y como Host- a espermatozoides con cola sin cambios (Jeyendran et al., 1984). En el caso de la viabilidad e integridad acrosomal, se consideron cuatro categorías: VA: espermatozoide vivo con acrosoma intacto; VS: espermatozoide vivo con acrosoma desprendido; MA: espermatozoide muerto con acrosoma intacto; MS: espermatozoide muerto con acrosoma desprendido (Didión et al., 1989).

\section{Análisis Estadístico}

Los datos de las variables motilidad, integridad de la membrana plásmatica y viablilidad e integridad acrosomal fueron transformados (arcoseno) para aproximar los valores a la distribución normal. En el experimento 1 se empleó el análisis de regresión polinomial quíntuple para determinar la concentración óptima del glicerol, etilenglicol y DMSO, respectivamente. En el experimento 2 se utilizó un análisis de varianza para evaluar el efecto del crioprotector sobre la motilidad, integridad de membrana plasmática y viabilidad e integridad acrosomal posdescongelamiento; además se realizó la prueba de Tukey para determinar cual de las concentraciones óptimas de los tres crioprotectores era la mejor. En todos los casos se empleó el paquete estadístico IBM SPSS Statistics 22.0 para Windows.

\section{Resultados}

Los resultados obtenidos de motilidad inicial y motilidad pos-descongelamiento con el glicerol, etilenglicol y DMSO para el experimento 1 se encuentran en el Cuadro 1. 
Cuadro 1. Motilidad inicial y pos-descongelamiento (promedio \pm d.e.) con ocho concentraciones de glicerol, etilenglicol y DMSO en espermatozoides epididimarios de alpaca

\begin{tabular}{lccc}
\hline Motilidad & Glicerol & Etilenglicol & DMSO \\
\hline Inicial & $66.0 \pm 8.9$ & $64.0 \pm 7.8$ & $70.0 \pm 7.1$ \\
Pos-descongelamiento & & & \\
T1 $(0 \mathrm{M})$ & $10.0 \pm 0$ & $5.4 \pm 3.2$ & $8.0 \pm 5.0$ \\
T2 $(0.25 \mathrm{M})$ & $22.0 \pm 13.1$ & $8.4 \pm 5.7$ & $12.3 \pm 4.1$ \\
T3 $(0.50 \mathrm{M})$ & $23.0 \pm 14.0$ & $9.0 \pm 3.6$ & $14.5 \pm 4.2$ \\
T4 $(0.75 \mathrm{M})$ & $26.0 \pm 15.2$ & $11.0 \pm 4.8$ & $22.5 \pm 7.9$ \\
T5 $(1.00 \mathrm{M})$ & $34.0 \pm 15.2$ & $12.0 \pm 3.9$ & $24.1 \pm 7.3$ \\
T6 $(1.25 \mathrm{M})$ & $36.0 \pm 15.2$ & $11.0 \pm 3.6$ & $15.0 \pm 5.6$ \\
T7 $(1.50 \mathrm{M})$ & $26.0 \pm 13.9$ & $9.4 \pm 3.3$ & $14.0 \pm 3.6$ \\
T8 $(1.75 \mathrm{M})$ & $22.0 \pm 13.0$ & $8.0 \pm 2.4$ & $12.8 \pm 5.2$ \\
\hline
\end{tabular}

Los valores son promedios \pm límite de confianza al 95\%

Cuadro 2. Efecto de la concentración optima del glicerol, etilenglicol y DMSO sobre las características espermáticas (promedio \pm d.e.) al inicio y después de la criopreservación de espermatozoides epididimarios de alpaca ( $\mathrm{n}=192$ pajillas)

\begin{tabular}{lccc}
\hline Motilidad & $\begin{array}{c}\text { Motilidad } \\
\text { espermática } \\
(\%)\end{array}$ & $\begin{array}{c}\text { Integridad } \\
\text { funcional de } \\
\text { membrana } \\
(\%)\end{array}$ & $\begin{array}{c}\text { Viabilidad e } \\
\text { integridad } \\
\text { acrosomal } \\
(\%)\end{array}$ \\
\hline Inicial & $67.5 \pm 7.5$ & $81.8 \pm 9.4$ & $75.8 \pm 8.9$ \\
Pos-descongelamiento & & & \\
T1 (Sin crioprotector) & $7.3 \pm 5.4^{\mathrm{c}}$ & $15.3 \pm 7.4^{\mathrm{c}}$ & $9.9 \pm 5.0^{\mathrm{c}}$ \\
T2 (1.16 M Gl) & $30.8 \pm 9.7^{\mathrm{a}}$ & $45.0 \pm 13.0^{\mathrm{a}}$ & $33.8 \pm 10.0^{\mathrm{a}}$ \\
T3 (1.02 M EG) & $17.4 \pm 12.2^{\mathrm{b}}$ & $32.2 \pm 14.1^{\mathrm{b}}$ & $21.0 \pm 7.5^{\mathrm{b}}$ \\
T4 (0.9 M DMSO) & $35.8 \pm 9.3^{\mathrm{a}}$ & $51.8 \pm 11.9^{\mathrm{a}}$ & $38.3 \pm 6.8^{\mathrm{a}}$ \\
\hline
\end{tabular}

$a, b, c$ Letras diferentes dentro de una columna indican diferencia significativa $(p<0.05)$

La Figura 1 muestra que las motilidades tienden a aumentar conforme se aumenta la concentración de glicerol hasta el valor de $1 \mathrm{M}$, para luego disminuir con concentraciones mayores. El análisis de regresión muestra que la pendiente es igual a cero $(\mathrm{R}=0.915)$ en la concentración de $1.16 \mathrm{M}$, por lo que esa sería la concentración óptima.
El etilenglicol presenta un comportamiento similar (Figura 2), donde la pendiente es igual a cero $(\mathrm{R}=0.740)$ en una concentración de $1.02 \mathrm{M}$, por lo que esa sería la concentración óptima. Asimismo, en el caso de DMSO, la motilidad aumenta hasta la concentración de $0.75 \mathrm{M}$ y comienzan a declinar con concentraciones mayores a $0.9 \mathrm{M}$ (Figura 


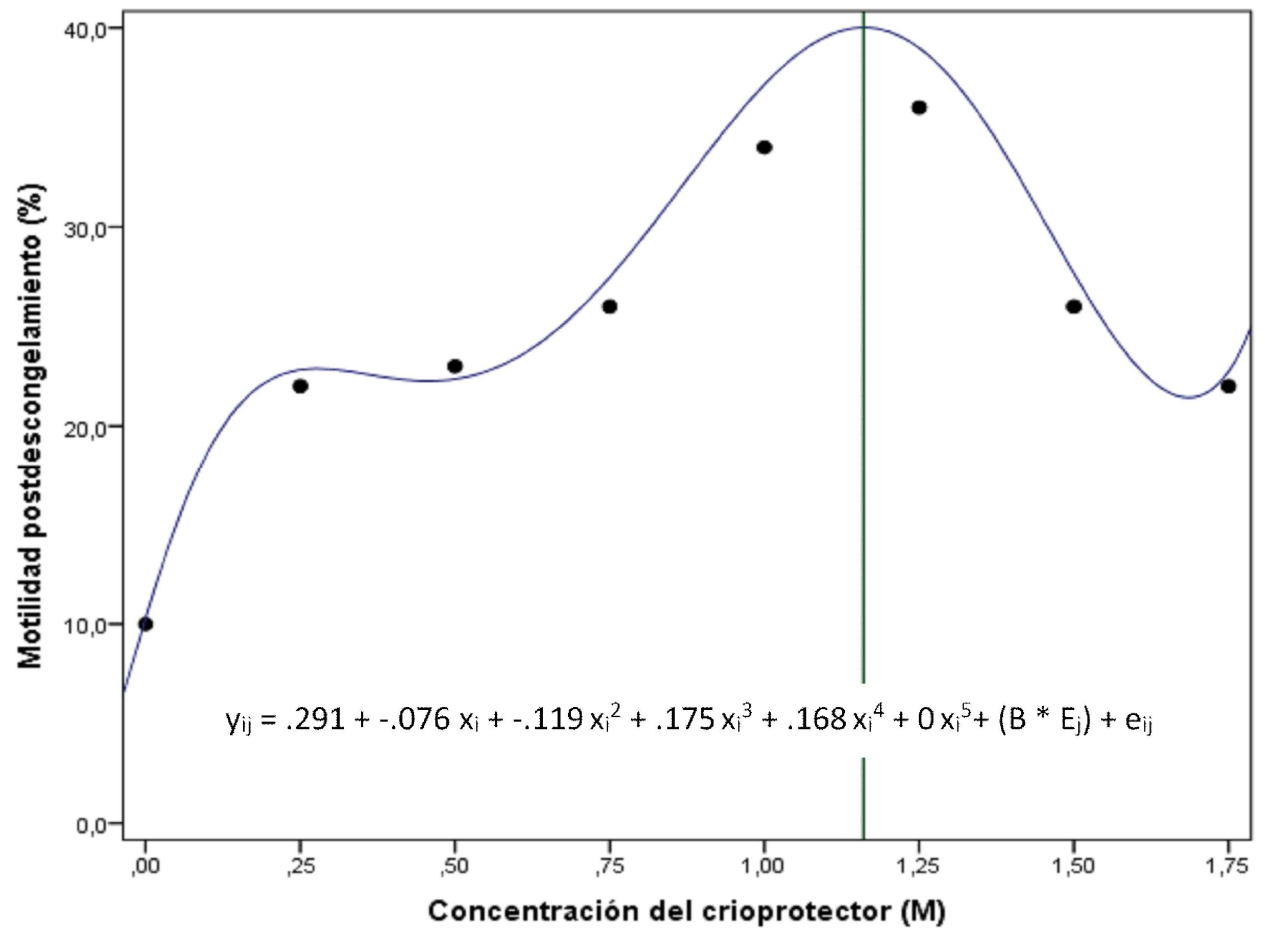

Figura 1. Diagrama de regresión polinomial quíntuple de la motilidad de espermatozoides epididimarios pos-descongelamiento con ocho concentraciones de glicerol

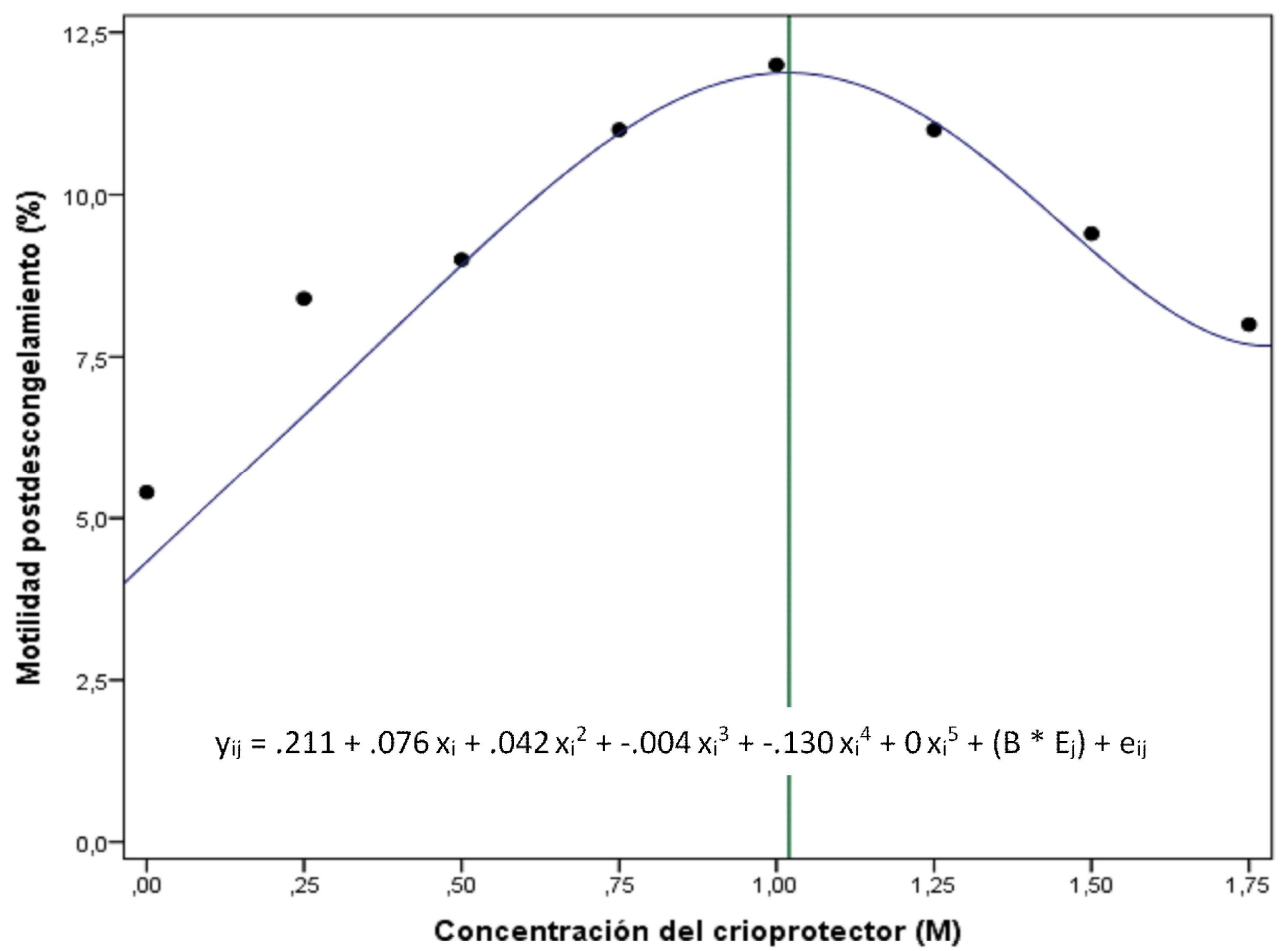

Figura 2. Diagrama de regresión polinomial quíntuple de la motilidad de espermatozoides epididimarios pos-descongelamiento con ocho concentraciones de etilenglicol 


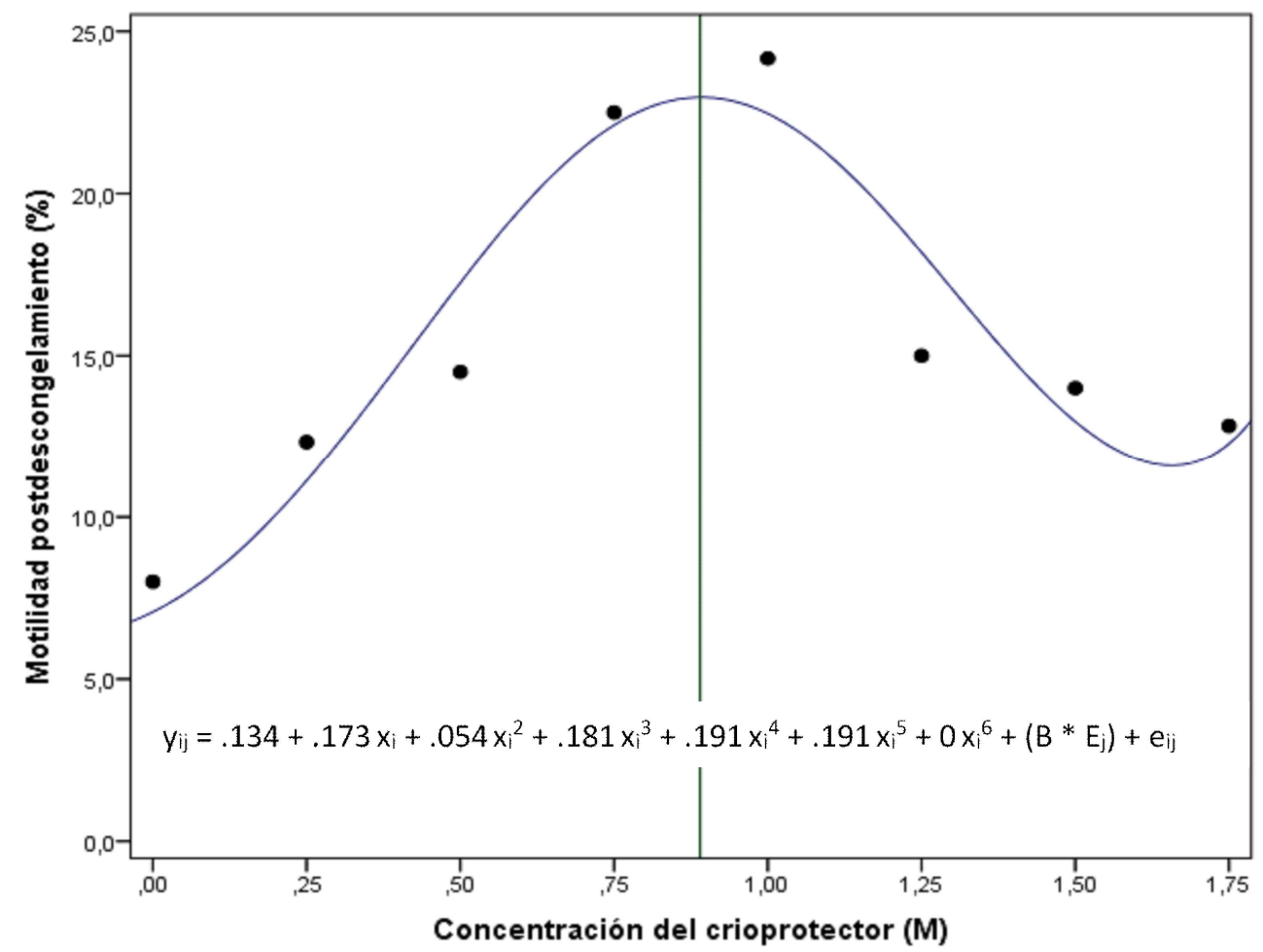

Figura 3. Diagrama de regresión polinomial quíntuple de la motilidad de espermatozoides epididimarios pos-descongelamiento con ocho concentraciones de dimetil sulfóxido (DMSO)

3); por otro lado, la pendiente es igual a cero $(\mathrm{R}=0.730)$ en una concentración de $0.9 \mathrm{M}$, por lo que esa sería la concentración óptima.

En el experimento 2, los mejores promedio pos-descongelamiento se observaron en T4 y T2 (Cuadro 2), sin deferencias significativas entre estos criopreservantes, aunque con promedios ligeramente superiores en T4.

\section{Discusión}

El tipo y la concentración del crioprotector influencia el proceso de criopreservación de los espermatozoides (Watson, 1990). En alpacas, el glicerol ha sido empleado en concentraciones de 7\% (0.96
M) (Santiani et al., 2005; Terreros et al., 2012), 6\% (0.82 M) (Banda et al., 2010) y 2 $4 \%(0.27-0.55 \mathrm{M})$ (Morton et al., 2007, 2010), obteniendo motilidades pos-descongelamiento entre 6 y $24 \%$, posiblemente por los efectos efectos tóxicos del glicerol (Garner et al., 1999). El etilenglicol se ha empleado al $1 \%(0.2 \mathrm{M})$ (Banda et al., 2010) y $7 \%(1.25 \mathrm{M})$ (Santiani et al., 2005; Terreros et al., 2012) con motilidades pos-descongelación entre 1 y $20 \%$. En estos estudios, Terreros et al. (2012) obtuvieron resultados inferiores que Santiani et al. (2005), posiblemente debido al tipo de muestra (espermatozoides epididimarios y semen eyaculado). Con el uso de DMSO al $0.9 \%(0.125 \mathrm{M}), 1.8 \%(0.25 \mathrm{M})$ (Canorio, 2008) y 7\% (0.98 M) se logró hasta $33 \%$ de motilidad pos-desconge-lamiento (Terreros et al., 2012). 
En los estudios antes mencionados no se pudo identificar el tipo y concentración ideal del crioprotector que permita obtener mejores tasas de motilidad pos-descongelamiento, lo que es de suma importancia, puesto que los crioprotectores son altamente especie específicos y su efectividad varía en función de la concentración utilizada (Gao et al., 1995). Al respecto, en el presente estudio se determinó que las concentraciones aparentemente óptimas del glicerol, etilenglicol y DMSO fueron de $1.16,1.02$ y $0.9 \mathrm{M}$, respectivamente, obteniéndose mayores tasas de motilidad para el glicerol (30.8\%) y DMSO $(35.8 \%)$, pero menores para el etilenglicol $(17.4 \%)$.

Los resultados del experimento 2 indicaron que el glicerol y DMSO presentaron mayores porcentajes de motilidad, integridad funcional de membrana plasmática y viabilidad e integridad acrosomal respecto al etilenglicol $(\mathrm{p}<0.05)$. Posiblemente, esto podría deberse al tiempo de exposición de los crioprotectores con los espermatozoides, dado que el etilenglicol, por su fácil penetración celular y congelación rápida puede ejercer efectos tóxicos mayores sobre la célula que los otros crioprotectores.

La motilidad es considerada como la principal característica al evaluar las técnicas de congelación, diluyentes y crioprotectores. Sin embargo, en la alpaca existe una gran variabilidad en los valores posdescongelamiento, tanto en el semen como en los espermatozoides epididimarios (Valdivia et al., 1999; Santiani et al., 2005; Morton et al., 2010; Banda et al., 2010; Terreros et al., 2012), lo que podría deberse a la motilidad inicial de las muestras; ya que al utilizar muestras con motilidades iniciales mayores a $60 \%$ se logran mejores porcentajes de motilidad después del descongelamiento (Choez et al., 2013).

La motilidad inicial en este estudio fue mayor de $60 \%$, obteniendo $31 \%$ de motilidad pos-descongelamiento para el glicerol; supe- rior al 15\% de Santiani et al. (2005), al 7$20 \%$ de Morton et al. $(2007,2010)$ y al $24 \%$ de Terreros et al. (2012), a pesar de haber utilizado un dilutor en base a leche descremada o lactosa. No obstante, fue similar al 32\% obtenido por Ordoñez y Verastegui (2007), quienes utilizaron el dilutor Tris con glicerol al 8\%. Por otro lado, el 36\% de motilidad obtenido con el DMSO fue similar a los reportes de Canorio (2008) y Terreros et al. (2012), aunque el primero utilizó un dilutor a base de citrato y en ambos casos se utilizón una menor concentración $(0.25 \mathrm{M})$, lo que indica que se pueden utilizar concentraciones más bajas de DMSO, si se utiliza citrato como dilutor.

El $17 \%$ de motilidad obtenida con etilenglicol fue similar a los resultados reportados por Banda et al. (2010), pero mayores al $8 \%$ alcanzado por Terreros et al. (2012) y $4 \%$ por Ordoñez y Verastegui (2007), quienes utilizaron concentraciones más altas de etilenglicol, lo que al parecer resultaron ser más tóxicas para el espermatozoide de alpaca.

Los porcentajes de integridad funcional de membrana plasmática del presente estudio de 45,32 y $52 \%$ para glicerol, etilenglicol y DMSO, respectivamente, fueron superiores a los valores de 24 y $17 \%$ reportados por Banda et al. (2010) utilizando glicerol y etilenglicol, respectivamente; asimismo, Terreros et al. (2012) reportaron porcentajes de 26,18 y $29 \%$ utilizando los tres crioprotectores del estudio, lo cual podría ocurrir debido a la técnica de congelación empleada de dos pasos, donde existe un mayor tiempo de exposición de los crioprotectores $\mathrm{y}$, por consiguiente, un mayor efecto sobre la membrana de los espermatozoides.

El 34, 21 y $38 \%$ de viabilidad e integridad acrosomal pos-descongelamiento para el glicerol, etilenglicol y DMSO, respectivamente, pueden considerarse como aceptables, en comparación con el estudio de Santiani et al. (2005), quienes obtuvieron tasas de 13 y $19 \%$ para el glicerol y etilenglicol. No obtante, 
Banda et al. (2010), usando glicerol y etilenglicol reportan valores de 33 y $27 \%$, respectivamente, y Morton et al. $(2007,2010)$ reportan valores hasta de $90 \%$ con glicerol.

Si bien, los resultados fueron alentadores; posiblemente el tiempo de transporte de los epidídimos hasta su procesamiento en el laboratorio, la velocidad de enfriamiento y el método de congelación empleado afectaron negativamente los resultados.

\section{Conclusiones}

- Las concentraciones de 0.9 M de DMSO y $1.16 \mathrm{M}$ de glicerol fueron más efectivas para conservar la motilidad, integridad funcional de la membrana plasmática $\mathrm{y}$ viabilidad e integridad de la membrana acrosomal de expermatozoides epididimarios de la alpaca por efecto de la criopreservación.

- El etilenglicol presentó una menor efectividad que el DMSO y el glicerol.

\section{Agradecimientos}

Los autores agradecen a Ricardo Castillo, Diego Evangelista, Javier Juárez y Gianfranco Arroyo por el apoyo prestado en el desarrollo del presente trabajo.

\section{Literatura Citada}

1. Ax RL, Dally M, Didion BA, Lenz $R W$, Love CC, Varner DD, et al. 2000. Semen evaluation. In: Hafez ESE, Hafez B (eds). Reproduction in farm animals. $7^{\text {th }}$ ed. Philadelphia, USA: Lippincott Williams \& Wilkins. p 365-375.

2. Banda J, Evangelista S, Ruiz L, Sandoval R, Rodríguez, C, Valdivia M, Santiani A. 2010. Efecto de dilutores en base a Tris, Tes y leche descremada en la criopreservación de espermatozoides obtenidos del epidídimo de alpa- ca. Rev Inv Vet Perú 21: 145-153. doi: 10.15381/rivep.v21i2.129

3. Bravo PW, Flores $U$, Garnica J, Ordonez C. 1997. Collection of semen and artificial insemination of alpacas. Theriogenology 47: 619-626. doi: 10.1016/S0093-691X(97)00020-4

4. Canorio N. 2008. Criocapacitación del espermatozoide de alpaca (Lama pacos). Tesis de Magíster. Lima: Univ Nacional Mayor de San Marcos. 102 p.

5. Choez K, Evangelista S, Castillo R, Santiani A. 2013. Efecto de la motilidad inicial y diferentes concentraciones de glicerol en la criopreservación de espermatozoides epididimarios de alpaca. Spermova 3: 83-84.

6. Didion B, Dobrinsky J, Giles J, Graves $C$. 1989. Staining procedure to defect viability and the true acrosome reaction in spermatozoa of various species. Gamete Res 22: 51-57. doi: 10.1002/mrd.1120220106

7. Gao DY, Liu J, Liu C, McGann LE, Watson PF, Kleinhans FW, Mazur P, et al. 1995. Prevention of osmotic injury to human spermatozoa during addition and removal of glycerol. Human Reprod 10: 1109-1122.

8. Garner DL, Thomas CA, Gravance CG 1999. The effect of glycerol on the viability, mitochondrial function and acrosomal integrity of bovine spermatozoa. Reprod Dom Anim 34: 399-404. doi: 10.1111/j.1439-0531.1999.tb01392.x

9. Hafez ESE. 2002. Preservación y criopreservación de gametos y embriones. En: Hafez ESE, Hafez B (eds). Reproducción e inseminación artificial en animales. $7^{\mathrm{a}}$ ed. México: McGraw Hill. p 441-452.

10. Jeyendran RS, van Der Ven HH, Perez-Pelaez M, Crabo BG, Zaneveld LJ. 1984. Development of an assay to assess the functional integrity of the human sperm membrane and its relationship to other semen characteristics. J Reprod Fertil 70: 219-228. doi: 10.1530/jrf.0.0700219 
11. Medeiros CMO, Forell F, Oliveira ATD, Rodriguez JL. 2002. Current status of cryopreservation: why isn't it better? Theriogenology 57: 327-344. doi: 10.1016/S0093-691X(01)00674-4

12. Morton K, Bathgate R, Evans G, Maxwell W. 2007. Cryopreservation of epididymal alpaca (Vicugna pacos) sperm: a comparison of citrate- Tris- and lactose- based diluents and pellets and straws. Reprod Fertil Dev 19: 792-796. doi: 10.1071/RD07049

13. Morton K, Evans G, Maxwell W. 2010. Effect of glycerol concentration, Equex STM ${ }^{\circledR}$ supplementation and liquid storage prior to freezing on the motility and acrosome integrity of frozen-thawed epididymal alpaca (Vicugna pacos) sperm. Theriogenology 74: 311-316. doi: 10.1016/j.theriogenology.2010.02.015

14. Ordoñez MJ, Verastegui HJ. 2007. Efecto de los crioprotectores (glicerol y etilenglicol) en la criopreservación de semen de alpaca (Lama pacos). Tesis de Ingeniero Zootecnista. Huancavelica: Univ Nacional de Huancavelica. 55 p.

15. Santiani A, Huanca W, Sapana $R$, Huanca T, Sepúlveda N, Sánchez R. 2005. Effects on the quality of frozen- thawed alpaca (Lama pacos) semen using two different cryoprotectants and extenders. Asian J Androl 7: 303-309. doi: 10.1111/j.1745-7262.2005.00021.x

16. Stornelli MC, Tittarelli CM, Savignone CA, Stornelli MA. 2005. Efecto de los procesos de criopreservación sobre ta fertilidad seminal. Analecta Vet 25: 28-35.

17. Terreros M, Huanca W, Arriaga I, Ampuero A. 2015. Efecto de tres crioprotectores en la criopreservación de espermatozoides epididimarios de alpaca. Rev Inv Vet Perú 26: 420-426. doi: 10.15381/rivep.v26i3.11182

18. Tibary A, Memon MA. 1999. Reproduction in the male South American Camelidae. J Camel Prac Res 6: 235-248.

19. Valdivia M, Ruiz M, Bermúdez L, Quinteros S, Gonzales A, Manosalva I, Ponce C, Olazábal J, Dávalos $R$. 1999. Criopreservación de semen de alpacas. Resumen II Congreso Mundial sobre Camelidos, Cusco, Perú.

20. Watson P. 1990. Artificial insemination and the preservation of semen. In: Marshall's phisiology of reproduction. $4^{\text {th }}$ ed. Edinbugh, UK. p 747-869. 